Advanced Computational Intelligence: An International Journal (ACII), Vol.3, No.2, April 2016

\title{
AN INTELLIGENT METHOD FOR ACCELERATING THE CONVERGENCE OF DIFFERENT VERSIONS OF SGMRES ALGORITHM
}

\author{
MohadesehEntezari Zarch ${ }^{1,2}$, ${ }^{*}$ SeyedAbolfazlShahzadeh Fazeli ${ }^{1,2}$, and Mohammad \\ Bagher Dowlatshahi ${ }^{1,3}$ \\ ${ }^{1}$ Parallel Processing Laboratory, Yazd University, Yazd,Iran. \\ ${ }^{2}$ Department of Computer Science, Faculty of Mathematics, Yazd \\ University, Yazd,Iran \\ ${ }^{3}$ Department of Computer Engineering, Faculty of Electronic and Computer, Yazd \\ University, Yazd,Iran.
}

\begin{abstract}
In a wide range of applications, solving the linear system of equations $A x=b$ is appeared. One of the best methods to solve the large sparse asymmetric linear systems is the simplified generalized minimal residual $(S G M R E S(m))$ method. Also, some improved versions of SGMRES $(m)$ exist: SGMRES-E $(m, k)$ and $S G M R E S-D R(m, k)$. In this paper, an intelligent heuristic method for accelerating the convergence of three methods SGMRES(m), SGMRES-E $(m, k)$, and SGMRES-DR( $m, k)$ is proposed. The numerical results obtained from implementation of the proposed approach on several University of Florida standard matrixes confirm the efficiency of the proposed method.
\end{abstract}

\section{Keywords}

Artificial Intelligence, Heuristic Algorithms, Linear systems of equations, SGMRES.

\section{INTRODUCTION}

In a wide range of applied sciences, solution of problems is obtained from solving a linear system of equations $\mathrm{Ax}=\mathrm{b}$. In numerical analysis, the algorithms for solving linear systems commonly use one of the two following methods: Direct methods, Iterative methods.

According to the coefficient of linear devices and matrix A, we can use one of these methods. If the matrix dimension is too small, direct methods such as Gaussian elimination method, GaussJordan and LU decomposition is preferred. These methods are composed of a finite number of steps. On the other hand, iterative methods are based on calculating a sequence of approximations to find the solution. In these methods, to stop the algorithm either an accurate solution is found or a certain number of iterations is performed. If the matrix A is relatively large, triangular-based direct methods are not recommended, because this method requires plenty of time and storage space. In addition, in many cases the coefficient matrix is sparse and triangular process destroys the sparseness of matrix, such we are faced with a large dense matrix. To solve such problems, it is recommended to use iterative methods which do not change the sparseness nature of matrix A. 
One of the main iterative methods, which nowadays has found many applications, is the generalized minimal residual method (GMRES) proposed by Saad and Schultz [1]. Simpler GMRES (SGMRES) method is a new version of GMRES, which was proposed by Walker and Zhou [2] and analyzed by Jiránek et al. [4]. SGMRES(m)) is a restarted version of SGMRES which restarts at the iteration when the Krylov subspace reaches dimension m, then the current solution is used as the new initial guess for the next $m$ iterations.

Despite the good performance of these methods, it seems that we can make changes in their structure to accelerate their convergence. In this study, an intelligent heuristic method to change the update equations of three methods SGMRES(m), SGMRES-E(m, k), and SGMRES-DR(m, k) is proposed. The experimental results on several University of Florida standard matrixes confirm the efficiency of the proposed method.

\section{THE SGMRES AND ITS VARIANTS}

The generalized minimal residual (GMRES) method developed by Saad and Schultz [1] is one of the most popular iterative methods for solving the large sparse nonsymmetrical system of linear equations $A x=b$, in which $A \in R^{n \times n}$ is nonsingular, $b \in R^{n}$ is a right-hand side vector, and $x \in R^{n}$ is the sought-after solution. Let $\mathrm{x}_{0} \in \mathrm{R}^{\mathrm{n}}$ be the initial guess, and $\mathrm{r}_{0}=\mathrm{b}-\mathrm{Ax} \mathrm{x}_{0}$ the initial residual vector. At the $\mathrm{m}^{\text {th }}$ iteration step, GMRES finds the approximate solution $\mathrm{x}_{\mathrm{m}}$ in the affine subspace $\mathrm{x}_{0}+\mathrm{K}_{\mathrm{m}}(\mathrm{A}$, $\mathrm{r}_{0}$ ), such that $\mathrm{x}_{\mathrm{m}}$ minimizes the Euclidean norm of the residual, i.e.:

$$
\begin{aligned}
& \left\|r_{m}\right\|=\left\|b-A x_{m}\right\|=\left\|b-A\left(x_{0}+z_{m}\right)\right\| \\
& \left.=\min _{z \in K_{m}\left(A, r_{0}\right)}\left\|b-A\left(x_{0}+z\right)\right\|=\min _{z \in K_{m}\left(A, r_{0}\right)} \| r_{0}-A z\right) \|
\end{aligned}
$$

Note that $K_{m}\left(A, r_{0}\right)=\operatorname{span}\left\{r_{0}, A r_{0}, \ldots, A^{m-1} r_{0}\right\}$ is the $m^{\text {th }}$ Krylov subspace constructed by the matrix $A$ and the initial residual vector $r_{0}$. It is obvious that (1) is corresponding to the orthogonality condition, i.e.:

$$
r_{m} \perp A K_{m}\left(A, r_{0}\right)
$$

where the orthogonality relation is established upon the Euclidean inner product.

The traditional implementation of GMRES is inspired from the Arnoldi process [3]. Application of $m$ steps of the Arnoldi process to the matrix $A$ with the nonzero residual vector $r_{0}$ yields the Arnoldi factorization

$$
A V_{m}=V_{m+1} \widehat{H}_{m}
$$

where the columns of the matrix $V_{m}$ form the orthonormal basis of the Krylov subspace $K_{\mathrm{m}}(A, r 0)$ and $\widehat{H}_{m} \in \mathrm{R}^{(m+1) \times m}$ is an upper Hessenberg matrix. We can reformulate the least-squares problem as the reduced minimization problem

$$
\left\|b-A x_{m}\right\|=\left\|b-A\left(x_{0}+V_{m} y_{m}\right)\right\|=\min _{y \in R^{m}}\left\|\beta e_{1}-\widehat{H}_{m} y\right\|
$$

where $\beta=\left\|r_{0}\right\|$ and $e_{1}$ is the first column of an identity matrix of order $m+1$. The $\mathrm{QR}$ factorization of $\widehat{H}_{m}$ with Givens rotations can be used to solve the reduced minimization problem. 
Simpler GMRES (SGMRES) method is a new version of GMRES, which was proposed by Walker and Zhou [2] and analyzed by Jiránek et al. [4]. In their method, instead of building an orthonormal basis of $K_{m}\left(A, r_{0}\right)$, an orthonormal basis $V_{m}$ of $A K_{m}\left(A, r_{0}\right)$ is established and then carries out the orthogonality relation (2). In a specific manner, suppose $Z_{m}=\left[z_{1}, z_{2}, \ldots, z_{m}\right]$ be a basis of $K_{m}\left(A, r_{0}\right)$. Then, the QR factorization of $\mathrm{AZ}_{\mathrm{m}}$ can be used to acquire the orthonormal basis $V_{m}$ of $A K_{m}\left(A, r_{0}\right)$, i.e.

$$
A Z_{m}=V_{m} R_{m}
$$

where $\mathrm{V}_{\mathrm{m}}=\left[\mathrm{v}_{1}, \mathrm{v}_{2}, \ldots, \mathrm{v}_{\mathrm{m}}\right]$ has orthonormal columns and $\mathrm{R}_{\mathrm{m}}$ is an $\mathrm{m} \times \mathrm{m}$ upper triangular and nonsingular matrix. By accomplishing the orthogonality relation (2), we can compute the $m^{\text {th }}$ residual vector $\mathrm{r}_{\mathrm{m}}$ recursively as

$$
\mathrm{r}_{\mathrm{m}}=\mathrm{r}_{0}-\mathrm{V}_{\mathrm{m}}\left(\mathrm{V}_{\mathrm{m}}^{\mathrm{T}} \mathrm{r}_{0}\right)=\mathrm{r}_{\mathrm{m}-1}-\alpha_{\mathrm{m}} \mathrm{v}_{\mathrm{m}} \quad \mathrm{m} \geq 1
$$

where $\alpha_{\mathrm{m}}=\mathrm{v}^{\mathrm{T}}{ }_{\mathrm{m}} \mathrm{r}_{0}$. The corresponding approximate solution is

$$
x_{m}=x_{0}+Z_{m} t_{m}
$$

where $t_{m}$ is the solution of the upper triangular system

$$
\mathrm{R}_{\mathrm{m}} \mathrm{t}_{\mathrm{m}}=\left[\alpha_{1}, \alpha_{2}, \ldots, \alpha_{\mathrm{m}}\right]^{\mathrm{T}} \text {. }
$$

By lapse of iterations, the amount of required computational time and space for GMRES or simpler GMRES increases meaningfully; and this subject makes GMRES and simpler GMRES impractical. In order to overcome this shortcoming, the restarted version of these algorithms is proposed. In restarted GMRES (GMRES(m)) or restarted simpler GMRES (SGMRES(m)), GMRES or SGMRES is restarted at the moment that the Krylov subspace reaches dimension $m$, then the current solution is used as the new initial guess for the next $m$ iterations. Some details of SGMRES(m) is presented in Algorithm 1[13].

Algorithm 1 (SGMRES(m)).

Input: $\mathrm{A}$ : the coefficient matrix; $\mathrm{b}$ : the right-hand side; $\mathrm{x}_{0}:$ an initial approximation; $\mathrm{m}:$ the maximal dimension of the Krylov subspace; $\varepsilon$ : a tolerance.

Output: An approximate solution x.

1. Compute $\mathrm{r}_{0}=\mathrm{b}-\mathrm{Ax} \mathrm{x}_{0}, \mathrm{z}_{1}=\mathrm{r}_{0} /\left\|\mathrm{r}_{0}\right\|, \hat{\mathrm{z}}_{1}=A \mathrm{z}_{1}, \mathrm{r}_{11}=\left\|\hat{\mathrm{z}}_{1}\right\|, \mathrm{v}_{1}=\hat{\mathrm{z}}_{1} / \mathrm{r}_{11}$

2. Compute $\alpha_{1}=v_{1}^{\mathrm{T}} \mathrm{r}_{0}, \mathrm{r}_{1}=\mathrm{r}_{0}-\alpha_{1} \mathrm{v}_{1}$

3. For $\mathrm{j}=2,3, \ldots, \mathrm{m}$

3.1. $z_{j}=v_{j-1}$

3.2. $\hat{z}_{\mathrm{j}}=A \mathrm{z}_{\mathrm{j}}$

3.3. For $\mathrm{i}=1,2, \ldots, \mathrm{j}-1$

End For

$$
\begin{aligned}
& r_{i j}=v_{i}^{T} \hat{z}_{j} \\
& \hat{z}_{j}=\hat{z}_{j}-r_{i j} v_{i}
\end{aligned}
$$

$$
\text { 3.4. } r_{i j}=\left\|\hat{z}_{j}\right\|, v_{j}=\hat{z}_{j} / r_{j j}, \alpha_{j}=v_{j}^{T} r_{j-1}
$$




$$
\text { 3.5. } r_{j}=r_{j-1}-\alpha_{j} v_{j}
$$

3.6. If $\left\|r_{j}\right\|<\varepsilon$ then solve the linear triangular system

$$
\mathrm{R}_{\mathrm{j}} \mathrm{t}=\alpha
$$

with $\alpha=\left[\alpha_{1}, \alpha_{2}, \ldots, \alpha_{j}\right]^{T}$ ، and form the approximate solutionx $x_{m}=x_{0}+z_{m}$ t and stop

4. Solve the linear triangular system

$$
\mathrm{R}_{\mathrm{j}} \mathrm{t}=\alpha
$$

With $\alpha=\left[\alpha_{1}, \alpha_{2}, \ldots, \alpha_{\mathrm{j}}\right]^{\mathrm{T}}$ and form the approximate solution $\mathrm{x}_{\mathrm{m}}=\mathrm{x}_{0}+\mathrm{Z}_{\mathrm{m}} \mathrm{t}$

5. Set $\mathrm{x}_{0}=\mathrm{x}_{\mathrm{m}}$ and go to Step 1 .

This implementation of SGMRES $(m)$ was proposed by Walker and Zhou [2]. In their implementation, they used $Z_{m}=\left[\mathrm{r}_{0} /\left\|\mathrm{r}_{0}\right\|, \mathrm{V}_{\mathrm{m}-1}\right]$. Note that different restarted simpler GMRES, called the residual-based restarted simpler GMRES (RB-SGMRES $(m)$ ), is proposed in [4]. RB$\operatorname{SGMRES}(m)$ uses

$$
\mathrm{Z}_{\mathrm{m}}=\left[\mathrm{r}_{0} /\left\|\mathrm{r}_{0}\right\|, \mathrm{r}_{1} /\left\|\mathrm{r}_{1}\right\|, \ldots, \mathrm{r}_{\mathrm{m}-1} /\left\|\mathrm{r}_{\mathrm{m}-1}\right\|\right]
$$

and is equivalent to SGMRES $(m)$. The implementation of RB-SGMRES $(m)$ is closely similar to that of SGMRES $(m)$ except that $z_{j}=r_{j-1} /\left\|r_{j-1}\right\|$ in Step 3.1 in Algorithm 1. In [4], it has been shown that $\operatorname{SGMRES}(m)$ is essentially unstable, but RB-SGMRES $(m)$ is conditionally stable provided that we have some reasonable residual decrease.

In general, because the Krylov subspace dimension is restricted at each cycle for the restarted methods, often, the convergence for the matrix with Ahaving small eigenvalues slows down. The main reason of this is that the Krylov subspace used at each cycle does not include good approximations to the eigenvectors corresponding to small eigenvalues. To accelerate the convergence of restarted GMRES, it is proposed to compute the approximate eigenvectors corresponding to small eigenvalues in modulus at each restart, and then augment the Krylov subspace by these approximate eigenvectors to improve the convergence of restarted GMRES. This kind of methods includes GMRES-E [5], GMRES-IR [6], and GMRES-DR [7]. These methods are equivalent at the end of each cycle. Although, GMRES-IR needs less matrix-vector products than GMRES-E at each cycle, it is slightly more complicated to implement. Moreover, GMRES-IR suffers from stability problems because it uses the implicitly restarted Arnoldi process [8], which may suffer from stability when a shift used in the implicitly restarted Arnoldi process is close to a true eigenvalue of the problem. GMRES-DR which is a good improvement of GMRES-IR has the efficiency of GMRES-IR, but is simpler to use and does not have the numerical stability problems [7].

Boojhawon and Bhuruth[9] by using augmentation technique applied the idea in GMRES-E to SGMRES, and proposed the simpler GMRES method augmented with approximate eigenvectors (SGMRES-E). The main advantage of SGMRES-E over GMRES-E is SGMRES-E requires a less computational time than GMRES-E.

Ref [13] improves the SGMRES-E by following the idea behind GMRES-DR and proposed the simpler GMRES method with deflated restarting (SGMRES-DR). SGMRES-DR includes the harmonic Ritz vectors corresponding to the smallest eigenvalues of the coefficient matrix $A$ at the 
Advanced Computational Intelligence: An International Journal (ACII), Vol.3, No.2, April 2016

beginning of each cycle. SGMRES-DR requires less matrix-vector products than SGMRES-E, and thus is more efficient.

\subsection{SGMRES with deflated restarting}

Suppose $\mathrm{W} \subset \mathrm{R}_{\mathrm{n}}$ be a subspace with dimension $\mathrm{m}$, the columns of $\mathrm{Z}_{\mathrm{m}}=\left[\mathrm{z}_{1}, \mathrm{z}_{2}, \ldots, \mathrm{z}_{\mathrm{m}}\right]$ be a basis of $\mathrm{W}, \mathrm{x}_{0} \in \mathrm{R}_{\mathrm{n}}$ be the initial guess, and $\mathrm{r}_{0}=\mathrm{b}-\mathrm{Ax} \mathrm{x}_{0}$ the initial residual vector. The purpose is to find the approximate solution $\mathrm{x}_{\mathrm{m}}$ in the affine subspace $\mathrm{x}_{0}+\mathrm{W}$ by imposing the minimization of the residual norm. It is equivalent to finding $\mathrm{x}_{\mathrm{m}}$ such that

$$
r_{m} \perp A W
$$

wherer $_{\mathrm{m}}=\mathrm{b}-\mathrm{Ax} \mathrm{x}_{\mathrm{m}}$, and $\mathrm{x}_{\mathrm{m}}=\mathrm{x}_{0}+\mathrm{Z}_{\mathrm{m}} \mathrm{y}_{\mathrm{m}}$ for some $\mathrm{y}_{\mathrm{m}} \in \mathrm{R}_{\mathrm{m}}$.

By applying the $\mathrm{QR}$ decomposition of $\mathrm{AZ}_{\mathrm{m}} \quad$ we have:

$$
A Z_{m}=V_{m} R_{m}(4)
$$

and applying the orthogonality relation (3), the expression of the $\mathrm{m}^{\text {th }}$ residual vector is obtained:

$$
\mathrm{r}_{\mathrm{m}}=\mathrm{r}_{0}-\mathrm{V}_{\mathrm{m}} \mathrm{V}_{\mathrm{m}}^{\mathrm{T}} \mathrm{r}_{0}=\mathrm{r}_{\mathrm{m}-1}-\mathrm{v}_{\mathrm{m}}\left(\mathrm{v}_{\mathrm{m}}^{\mathrm{T}} \mathrm{r}_{0}\right)
$$

The harmonic Ritz vectors corresponding to the smallest eigenvalues of A is used in SGMRESDR because they are better approximate eigenvectors for eigenvalues with small modulus [10]. Note that the term "the smallest eigenvalues" means the smallest eigenvalues in modulus. The harmonic Ritz vector $\mathrm{u}=\mathrm{Z}_{\mathrm{m}} \mathrm{y}$ satisfies the following orthogonality condition

which is equivalent to

$$
(\mathrm{Au}-\lambda \mathrm{u}) \perp \mathrm{AZ}_{\mathrm{m}}
$$

i.e.,

$$
\mathrm{V}_{\mathrm{m}}^{\mathrm{T}}\left(\mathrm{AZ} \mathrm{m} \mathrm{y}-\lambda \mathrm{Z}_{\mathrm{m}} \mathrm{y}\right)=0
$$

$$
\mathrm{R}_{\mathrm{m}} \mathrm{y}=\lambda \mathrm{V}_{\mathrm{m}}^{\mathrm{T}} \mathrm{Z}_{\mathrm{m}} \mathrm{Y}
$$

Suppose $\mathrm{y}_{1}, \mathrm{y}_{2}, \ldots, \mathrm{y}_{\mathrm{k}}$ are $\mathrm{k}$ eigenvectors corresponding to $\mathrm{k}$ smallest eigenvalues of the reduced generalized eigenvalue problem (5).

In the SGMRES-E, proposed by Boojhawon and Bhuruth in [9], approximate eigenvectors used to augment the Krylov subspaces are harmonic Ritz vectors $\hat{x}_{j}=Z_{m} y_{j}, j=1,2, \ldots, k$. The implementation of SGMRES-E is outlined in Algorithm 2[13].

\section{Algorithm 2(SGMRES-E(m, k)).}

Input: $\mathrm{A}$ : the coefficient matrix; $\mathrm{b}:$ the right-hand side; $\mathrm{x}_{0}$ : an initial approximation; $\mathrm{m}:$ the maximal dimension of the Krylov subspace; $\mathrm{k}$ : the number of harmonic Ritz vectors; $\varepsilon$ : a tolerance.

Output:An approximate solution x. 
1. Apply one cycle of SGMRES(m) to generate $Z_{m}, V_{m}, R_{m}, x_{m}$, and $r_{m}$.

2. Compute the eigenvalues and eigenvectors of the generalized eigenvalue problem (6) by the QZ algorithm [11]. Let $\mathrm{y}_{1}, \mathrm{y}_{2}, \ldots, \mathrm{y}_{\mathrm{k}}$ be $\mathrm{k}$ eigenvectors corresponding to $\mathrm{k}$ smallest eigenvalues of (6).

3. Form $\mathrm{k}$ harmonic Ritz vectors $\hat{\mathrm{x}}_{\mathrm{j}}=\mathrm{Z}_{\mathrm{m}} \mathrm{y}_{\mathrm{j}}, \mathrm{j}=1,2, \ldots, \mathrm{k}$

4. Set $\mathrm{x} 0=\mathrm{x}_{\mathrm{m}}$, and compute $\mathrm{r}_{0}=\mathrm{r}_{\mathrm{m}}, \mathrm{z}_{1}=\mathrm{r}_{0} /\left\|\mathrm{r}_{0}\right\|, \hat{\mathrm{z}}_{1}=A \mathrm{z}_{1}, \mathrm{r}_{11}=\left\|\hat{\mathrm{z}}_{1}\right\|, \mathrm{v}_{1}=\hat{\mathrm{z}}_{1} / \mathrm{r}_{11}$

5. Compute $\alpha_{1}=\mathrm{v}_{1}^{\mathrm{T}} \mathrm{r}_{0}, \mathrm{r}_{1}=\mathrm{r}_{0}-\alpha_{1} \mathrm{v}_{1}$

6. For $\mathrm{j}=2,3, \ldots, \mathrm{m}$

6.1. If $\mathrm{j} \leq \mathrm{m}-$ kthen $\mathrm{z}_{\mathrm{j}}=\mathrm{v}_{\mathrm{j}-1}$ else $\mathrm{z}_{\mathrm{j}}=\hat{\mathrm{x}}_{\mathrm{j}-\mathrm{m}+\mathrm{k}}$

6.2. $\hat{z}_{\mathrm{j}}=A \mathrm{z}_{\mathrm{j}}$

6.3. For $\mathrm{i}=1,2, \ldots, \mathrm{j}-1$

$\mathrm{r}_{\mathrm{ij}}=\mathrm{v}_{\mathrm{i}}^{\mathrm{T}} \hat{\mathrm{z}}_{\mathrm{j}} \hat{\mathrm{z}}_{\mathrm{j}}=\hat{\mathrm{z}}_{\mathrm{j}}-\mathrm{r}_{\mathrm{ij}} \mathrm{v}_{\mathrm{i}}$

End For

6.4. $r_{j j}=\left\|\hat{z}_{j}\right\|, v_{j}=\hat{z}_{j} / r_{j j}, \alpha_{j}=v_{j}^{T} r_{j-1}$

6.5. $r_{j}=r_{j-1}-\alpha_{j} v_{j}$

6.6. If $\left\|\mathrm{r}_{\mathrm{j}}\right\|<\varepsilon$ then solve the linear triangular system

$$
\mathrm{R}_{\mathrm{j}} \mathrm{t}=\alpha
$$

with $\alpha=\left[0, \ldots, 0, \alpha_{\mathrm{k}+1}, \ldots, \alpha_{\mathrm{j}}\right]^{\mathrm{T}}$ ، and form the approximate solution $\mathrm{x}=\mathrm{x}_{0}+\mathrm{Z}_{\mathrm{j}} \mathrm{t}$, and stop.

7. Solve the linear triangular systemR $\mathrm{R}_{\mathrm{m}} \mathrm{t}=\alpha$ with $\alpha=\left[\alpha_{1}, \alpha_{2}, \ldots, \alpha_{\mathrm{j}}\right]^{\mathrm{T}}$ and form the approximate solution $\mathrm{x}_{\mathrm{m}}=\mathrm{x}_{0}+\mathrm{Z}_{\mathrm{m}}$ t. Go to Step 2 .

Suppose $\mathrm{Y}_{\mathrm{k}}=\left[\mathrm{y}_{1}, \mathrm{y}_{2}, \ldots, \mathrm{y}_{\mathrm{k}}\right]$ and let $\mathrm{P}_{\mathrm{k}} \mathrm{L}_{\mathrm{k}}=\mathrm{Y}_{\mathrm{k}}$ be the $\mathrm{QR}$ decomposition of $\mathrm{Y}_{\mathrm{k}}$. Multiplying (4) by $\mathrm{P}_{\mathrm{k}}$ from the right yields

$$
A Z_{m} P_{k}=V_{m} R_{m} P_{k}
$$

Let $\mathrm{Q}_{\mathrm{k}} \mathrm{R}_{\mathrm{k}}^{\text {new }}=\mathrm{R}_{\mathrm{m}} \mathrm{P}_{\mathrm{k}}$ be the $\mathrm{QR}$ decomposition of $\mathrm{R}_{\mathrm{m}} \mathrm{P}_{\mathrm{k}}$. From the above equality, we obtain

Define

$$
\mathrm{AZ}_{\mathrm{m}} \mathrm{P}_{\mathrm{k}}=\mathrm{V}_{\mathrm{m}} \mathrm{Q}_{\mathrm{k}} \mathrm{R}_{\mathrm{k}}^{\text {new }}
$$

So, we have

$$
\mathrm{Z}_{\mathrm{k}}^{\text {new }}=\mathrm{Z}_{\mathrm{m}} \mathrm{P}_{\mathrm{k}}, \quad \mathrm{V}_{\mathrm{k}}^{\text {new }}=\mathrm{V}_{\mathrm{m}} \mathrm{Q}_{\mathrm{k}}
$$

$$
A Z_{\mathrm{k}}^{\text {new }}=\mathrm{V}_{\mathrm{k}}^{\text {new }} \mathrm{R}_{\mathrm{k}}^{\text {new }}
$$

where $\mathrm{Z}_{\mathrm{k}}^{\text {new }}, \mathrm{V}_{\mathrm{k}}^{\text {new }} \in \mathrm{R}^{\mathrm{n} \times \mathrm{k}}$, and $\mathrm{V}_{\mathrm{k}}^{\text {new }}$ has orthonormal columns, $R_{k}^{\text {new }} \in R^{k \times k}$ is an upper triangular matrix. This is $\mathrm{aQR}$ decomposition with $\mathrm{k}$ new vectors.We now restart the GMRES method. Note that

$$
\mathrm{x}_{0}^{\text {new }}=\mathrm{x}_{\mathrm{m}}, \mathrm{r}_{0}^{\text {new }}=\mathrm{r}_{\mathrm{m}}=\mathrm{r}_{0}-\mathrm{V}_{\mathrm{m}} \mathrm{V}_{\mathrm{m}}^{\mathrm{T}} \mathrm{r}_{0}
$$

Therefore, the $k^{\text {th }}$ residual vector at the new cycle is

Since

$$
\mathrm{r}_{\mathrm{k}}^{\text {new }}=\mathrm{r}_{0}^{\text {new }}-\mathrm{V}_{\mathrm{k}}^{\text {new }}\left(\left(\mathrm{V}_{\mathrm{k}}^{\text {new }}\right)^{\mathrm{T}} \mathrm{r}_{0}^{\text {new }}\right)
$$

$$
\left(V_{k}^{\text {new }}\right)^{\mathrm{T}} \mathrm{r}_{0}^{\text {new }}=\mathrm{Q}_{\mathrm{k}}^{\mathrm{T}} \mathrm{V}_{\mathrm{m}}^{\mathrm{T}} \mathrm{r}_{\mathrm{m}}=\mathrm{Q}_{\mathrm{k}}^{\mathrm{T}} \mathrm{V}_{\mathrm{m}}^{\mathrm{T}}\left(\mathrm{r}_{0}-\mathrm{V}_{\mathrm{m}} \mathrm{V}_{\mathrm{m}}^{\mathrm{T}} \mathrm{r}_{0}\right)=0
$$


Advanced Computational Intelligence: An International Journal (ACII), Vol.3, No.2, April 2016

we obtain

$$
\mathrm{r}_{\mathrm{k}}^{\text {new }}=\mathrm{r}_{0}^{\text {new }}
$$

to extend the new QR factorization of order $k$ (7) to obtain a $\mathrm{QR}$ factorization of order $m$, i.e., a QR factorization with $m$ vectors.

In SGMRES-DR, we have

$$
z_{j+1}^{\text {new }} \cdot j=k, k+1, \ldots, m-1 a s z_{j+1}^{n e w}=v_{j}^{\text {new }}
$$

Then, the expansion of (7) to a QR factorization of order $m$ can be done as follows: the vector $A z_{k+1}^{\text {new }}=A v_{k}^{\text {new }}$ is orthogonalized against $V_{k}^{\text {new }}$ and normalized to give $v_{k+1}^{\text {new }}$, after which $R_{k+1}^{\text {new }}$ is formed from $R_{k}^{\text {new }}$. With $z_{j+1}^{\text {new }}=v_{j}^{\text {new }}, j=k, k+1, \ldots, m-1$, the process is iterated until a $Q R$ factorization of order $\mathrm{m}$ is obtained. The implementation of SGMRES-DR is outlined in Algorithm 3[13].

Algorithm 3 (SGMRES-DR(m, k)).

Input: $\mathrm{A}$ : the coefficient matrix; $\mathrm{b}$ : the right-hand side; $\mathrm{x}_{0}:$ an initial approximation; $\mathrm{m}:$ the maximal dimension of the Krylov subspace; $\mathrm{k}$ : the number of harmonic Ritz vectors; $\varepsilon: \mathrm{a}$ tolerance.

Output: An approximate solution x.

1. Apply one cycle of SGMRES(m) to generate $Z_{m}, V_{m}, R_{m}, x_{m}$, and $r_{m}$.

2. Compute the eigenvalues and eigenvectors of the generalized eigenvalue problem (6) by the QZ algorithm [11]. Set $\mathrm{Y}_{\mathrm{k}}=\left[\mathrm{y}_{1}, \mathrm{y}_{2}, \ldots, \mathrm{y}_{\mathrm{k}}\right]$, where $\mathrm{y}_{1}, \mathrm{y}_{2}, \ldots, \mathrm{y}_{\mathrm{k}}$ are $\mathrm{k}$ eigenvectors corresponding to $\mathrm{k}$ smallest eigenvalues of (6).

3. Compute the $\mathrm{QR}$ decomposition of $\mathrm{Y}_{\mathrm{k}}: \mathrm{P}_{\mathrm{k}} \mathrm{L}_{\mathrm{k}}=\mathrm{Y}_{\mathrm{k}}$ and the $\mathrm{QR}$ decomposition of $\mathrm{R}_{\mathrm{m}} \mathrm{P}_{\mathrm{k}}: \mathrm{Q}_{\mathrm{k}} \mathrm{R}_{\mathrm{k}}^{\text {new }}=\mathrm{R}_{\mathrm{m}} \mathrm{P}_{\mathrm{k}}$.

4. $\quad$ Set $Z_{k}^{\text {new }}=Z_{m} P_{k}$ and $V_{k}^{\text {new }}=V_{m} Q_{k}$.

5. Let $Z_{k}=Z_{k}^{\text {new }}, V_{k}=V_{k}^{\text {new }}, R_{k}=R_{k}^{\text {new }}, x_{0}=x_{m}, r_{0}=r_{m}, r_{k}=r_{0}$

6. For $\mathrm{j}=\mathrm{k}+1, \mathrm{k}+2, \ldots, \mathrm{m}$

6.1. $\mathrm{z}_{\mathrm{j}}=\mathrm{v}_{\mathrm{j}-1}$

6.2. $\hat{z}_{\mathrm{j}}=\mathrm{Az}$

6.3. For $\mathrm{i}=1,2, \ldots, \mathrm{j}-1$

$\mathrm{r}_{\mathrm{ij}}=\mathrm{v}_{\mathrm{i}}^{\mathrm{T}} \hat{\mathrm{z}}_{\mathrm{j}} \hat{\mathrm{z}}_{\mathrm{j}}=\hat{\mathrm{z}}_{\mathrm{j}}-\mathrm{r}_{\mathrm{ij}} \mathrm{v}_{\mathrm{i}}$

End For

6.4. $r_{j j}=\left\|\hat{z}_{j}\right\|, v_{j}=\hat{z}_{j} / r_{j j}, \alpha_{j}=v_{j}^{T} r_{j-1}$

6.5. $r_{j}=r_{j-1}-\alpha_{j} v_{j}$

6.6. If $\left\|\mathrm{r}_{\mathrm{j}}\right\|<\varepsilon$, then solve the linear triangular system

$\mathrm{R}_{\mathrm{j}} \mathrm{t}=\alpha$

With $\alpha=\left[0, \ldots, 0, \alpha_{\mathrm{k}+1}, \ldots, \alpha_{\mathrm{m}}\right]^{\mathrm{T}}$, and form the approximate solution $\mathrm{x}_{\mathrm{m}}=\mathrm{x}_{0}+\mathrm{Z}_{\mathrm{m}} \mathrm{t}$.

Go to Step 2. 
If the eigenvector $\mathrm{y}_{\mathrm{i}}$ at Step 2 in Algorithm 3 is complex, the matrix $Y_{\mathrm{k}}$ should include both the real and imaginary parts of $y_{\mathrm{i}}$. In this situation, we may have to tune $\mathrm{k}$ eigenvectors at a restart.

After running one cycle of SGMRES(m), we reach toZ $Z_{m}=\left[r_{0} /\left\|r_{0}\right\|, V_{m-1}\right]$. Based on [12], the matrix $\mathrm{V}_{\mathrm{m}}^{\mathrm{T}} \mathrm{Z}_{\mathrm{m}}$ in the generalized eigenvalue problem (5) can be formulated as

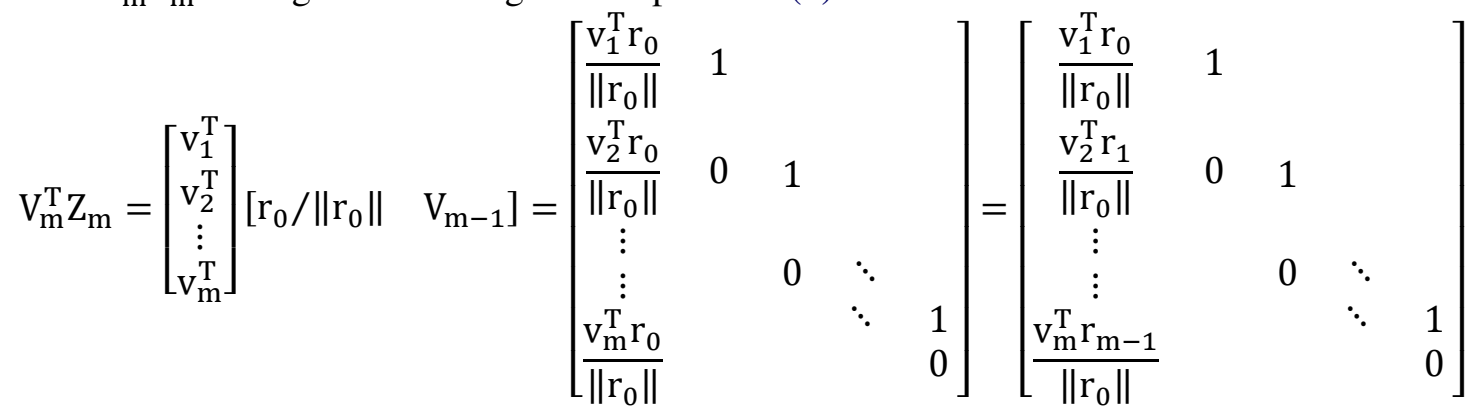

Here, the next identity: for all $j>i, v_{j}^{T} r_{i}=v_{j}^{T} r_{j-1}$, has been used. Becausev $v_{j}^{T} r_{j-1}, j=1,2, \ldots m$, has been generated in applying one cycle of SGMRES $(\mathrm{m})$, the matrix $\mathrm{V}_{\mathrm{m}}^{\mathrm{T}} \mathrm{Z}_{\mathrm{m}}$ without additional inner products of vectors can be formed with order $n$.

For other cycles, we have $\quad \mathrm{Z}_{\mathrm{m}}=\left[\mathrm{z}_{1}, \ldots, \mathrm{z}_{\mathrm{k}}, \mathrm{v}_{\mathrm{k}}, \ldots, \mathrm{v}_{\mathrm{m}-1}\right]$

So, $\quad \mathrm{v}_{\mathrm{m}}^{\mathrm{T}} \mathrm{Z}_{\mathrm{m}}=\left[\begin{array}{c}\mathrm{v}_{1}^{\mathrm{T}} \\ \vdots \\ \mathrm{v}_{\mathrm{k}}^{\mathrm{T}} \\ \mathrm{v}_{\mathrm{k}+1}^{\mathrm{T}} \\ \vdots \\ \mathrm{v}_{\mathrm{m}}^{\mathrm{T}}\end{array}\right]\left[\mathrm{z}_{1}, \ldots, \mathrm{z}_{\mathrm{k}}, \mathrm{v}_{\mathrm{k}}, \ldots, \mathrm{v}_{\mathrm{m}-1}\right]=\left[\begin{array}{ccccccc}\mathrm{v}_{1}^{\mathrm{T}} \mathrm{z}_{1} & \cdots & \mathrm{v}_{1}^{\mathrm{T}} \mathrm{z}_{\mathrm{k}} & & & & \\ \vdots & \vdots & \vdots & & & & \\ \mathrm{v}_{\mathrm{k}}^{\mathrm{T}} \mathrm{z}_{1} & \vdots & \mathrm{v}_{\mathrm{k}}^{\mathrm{T}} \mathrm{z}_{\mathrm{k}} & 1 & & & \\ \mathrm{v}_{\mathrm{k}+1}^{\mathrm{T}} \mathrm{z}_{1} & \vdots & \mathrm{v}_{\mathrm{k}+1}^{\mathrm{T}} \mathrm{z}_{\mathrm{k}} & 0 & 1 & & \\ \mathrm{v}_{\mathrm{k}+2}^{\mathrm{T}} \mathrm{z}_{1} & \vdots & \mathrm{v}_{\mathrm{k}+2}^{\mathrm{T}} \mathrm{z}_{\mathrm{k}} & & 0 & \ddots & \\ \vdots & \vdots & \vdots & & & \ddots & 1 \\ \mathrm{v}_{\mathrm{m}}^{\mathrm{T}} \mathrm{z}_{1} & \cdots & \mathrm{v}_{\mathrm{m}}^{\mathrm{T}} \mathrm{z}_{\mathrm{k}} & & & & 0\end{array}\right]$

Thus, $\mathrm{km}$ additional inner products of vectors with order $\mathrm{n}$ to form $\mathrm{V}_{\mathrm{m}}^{\mathrm{T}} \mathrm{Z}_{\mathrm{m}}$ is required.

\section{THE PROPOSED METHOD}

As mentioned in the previous section, methods for solving linear system $\mathrm{Ax}=\mathrm{b}$ start with an initial vector $x$, and iteratively try to change the values of these vectors in order to reduce the estimation error. In this methods, unfortunately, the process of calculating the amount of change of the vector $\mathrm{x}$ is a time consuming process. Therefore, in this paper, we will use an intelligent heuristic method to quickly predict the amount of change of the vector $\mathrm{x}$ in each iteration. The proposed heuristic is as follows: "The value of each dimension $\mathrm{d}$ of vector $\mathrm{x}$ which in c previous iterations of the algorithm steadily decreases (increases) is likely to decrease (increase) further in the next iteration." So, without much calculation, we can perform intelligent changes in the direction of each dimension of vector $\mathrm{x}$. By this assumption, in practice dramatic convergence will be reached.

By changing the step 6 of algorithm 1, the Improved SGMRES(m) (ISGMRES(m)) is obtained. The implementation of ISGMRES(m) is outlined in Algorithm 4. 
Advanced Computational Intelligence: An International Journal (ACII), Vol.3, No.2, April 2016

Algorithm 4:The template of ISGMRES(m)

1. Step 1 to step 5 of algorithm 1.

2. Decrease (increase) the value of each dimension $d$ of vector $x$ which in $c$ previous iterations of the algorithm steadily decrease (increase), by $\beta * x^{\prime}(d)$, where $\beta$ is a real number that is called the update rate and $x^{\prime}(d)$ is the amount of change in the value of vector xin dimension $\mathrm{d}$ in the before iteration.

3. Goto step 1.

By changing the step 8 of algorithm 2, the Improved SGMRES-E(m,k) (ISGMRES-E(m,k)) can be obtained. The implementation of ISGMRES-E $(\mathrm{m}, \mathrm{k})$ is outlined in Algorithm 5.

Algorithm 5: The template of ISGMRES-E $(\mathrm{m}, \mathrm{k})$

1. Step 1 to step 7 of algorithm 2.

2. Decrease (increase) the value of each dimension $d$ of vector $x$ which in $c$ previous iterations of the algorithm steadily decrease (increase), by $\beta * x^{\prime}(d)$, where $\beta$ is a real number that is called the update rate and $x^{\prime}(d)$ is the amount of change in the value of vector xin dimension $\mathrm{d}$ in the before iteration.

3. Goto step 1.

By changing the step 8 of algorithm 3, the Improved SGMRES-DR(m,k) (ISGMRES-DR(m,k)) is obtained. The implementation of ISGMRES-DR $(\mathrm{m}, \mathrm{k})$ is outlined in Algorithm 6.

Algorithm 6: The template of ISGMRES-E(m,k)

1. Step 1 to step 7 of algorithm 3.

2. Decrease (increase) the value of each dimension $d$ of vector $x$ which in $c$ previous iterations of the algorithm steadily decrease (increase), by $\beta * x^{\prime}(d)$, where $\beta$ is a real number that is called the update rate and $x^{\prime}(d)$ is the amount of change in the value of vector xin dimension $\mathrm{d}$ in the before iteration.

3. Goto step 1.

\section{Experimental results}

To investigate the performance of proposed methods, several experiments on a number of standard matrices of the University of Florida has been carried out. In all these experiments, the vector $\mathrm{x}_{0}$ is initialized by zero, and computational error $\|b-A * x\| /\|b\|$ intended. In Table 1, SGMRES(m) and ISGMRES(m) are compared. According to Table 1, Figure1, and Figure 2, the performance of ISGMRES(m) is better than SGMRES(m).

Table 1: Comparison of the results of SGMRES(m) and ISGMRES(m)

\begin{tabular}{ccccccc}
\hline Problem & m & error & SGMRES & \multicolumn{3}{c}{ ISGMRES } \\
\hline & & & iteration & c & $\beta$ & iteration \\
Tols1090 & 20 & 0.2375 & 200 & 10 & 0.05 & $\mathbf{1 4 4}$ \\
Tols1090 & 20 & 0.1847 & 500 & 10 & 0.007 & $\mathbf{4 5 8}$ \\
Zenios & 30 & 0.9585 & 200 & 5 & 0.00006 & $\mathbf{8 4}$ \\
Zenios & 30 & 0.9584 & 500 & 5 & 0.00007 & $\mathbf{2 5 2}$ \\
Qh148 & 20 & 0.9898 & 200 & 5 & 0.005 & $\mathbf{2 0 0}$ \\
Qh148 & 20 & 0.9898 & 500 & 5 & 0.9 & $\mathbf{2 5 7}$ \\
\hline
\end{tabular}


Advanced Computational Intelligence: An International Journal (ACII), Vol.3, No.2, April 2016

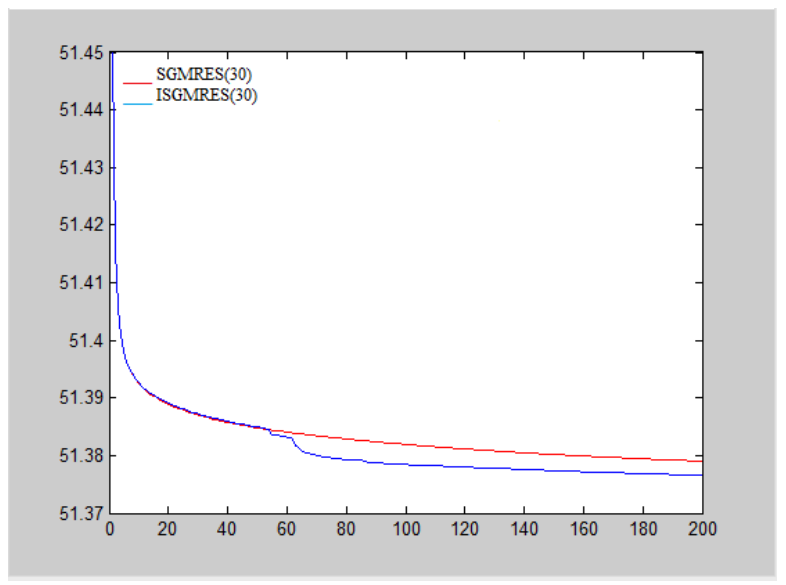

Figure 1: Comparison of convergence of SGMRES(m) and ISGMRES(m) on Zenios matrix with up to 200 iteration

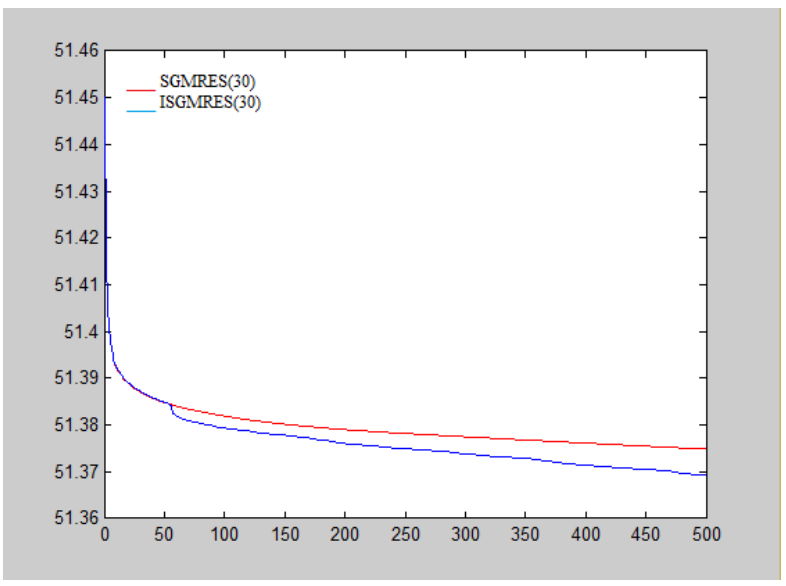

Figure 2: Comparison of convergence of SGMRES(m) and ISGMRES(m) on Zenios matrix with up to 500 iteration

In Table 2, SGMRES-E(m,k) and ISGMRES-E(m,k) is compared. According to Table 2,Figure3, and Figure 4, the performance of ISGMRES-E $(m, k)$ is better than SGMRES-E $(m, k)$.

Table 2: Comparison of the results of SGMRES-E(m,k) and ISGMRES-E(m,k)

\begin{tabular}{cccccccc}
\hline Problem & m & $\mathbf{k}$ & error & $\begin{array}{c}\text { SGMRES- } \\
\text { E }\end{array}$ & \multicolumn{3}{c}{ ISGMRES-E } \\
\hline Tols1090 & 20 & 5 & 0.2603 & 200 & 20 & 0.8 & iteration \\
Tols1090 & 20 & 5 & 0.2063 & 500 & 20 & 0.4 & $\mathbf{4 0 8}$ \\
Zenios & 30 & 10 & 0.9574 & 200 & 5 & 0.00005 & $\mathbf{1 8 2}$ \\
Zenios & 30 & 10 & 0.9570 & 500 & 10 & 0.0005 & $\mathbf{5 0 0}$ \\
Qh148 & 20 & 5 & 0.9758 & 200 & 1 & 0.006 & $\mathbf{1 9 0}$ \\
Qh148 & 20 & 5 & 0.9750 & 500 & 15 & 0.1 & $\mathbf{5 0 0}$ \\
\hline
\end{tabular}


Advanced Computational Intelligence: An International Journal (ACII), Vol.3, No.2, April 2016

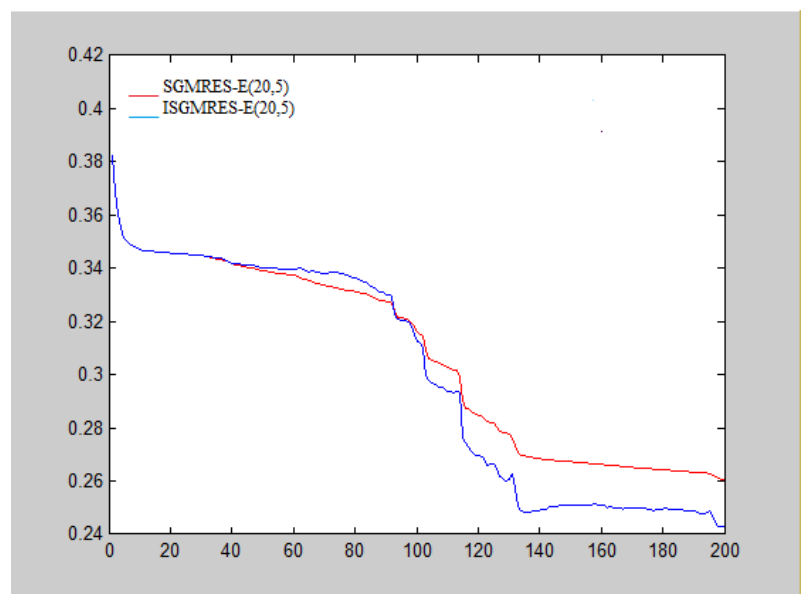

Figure 3: Comparison of convergence of SGMRES-E(m,k) and ISGMRES-E(m,k) on Tols1090 matrix with up to 200 iteration

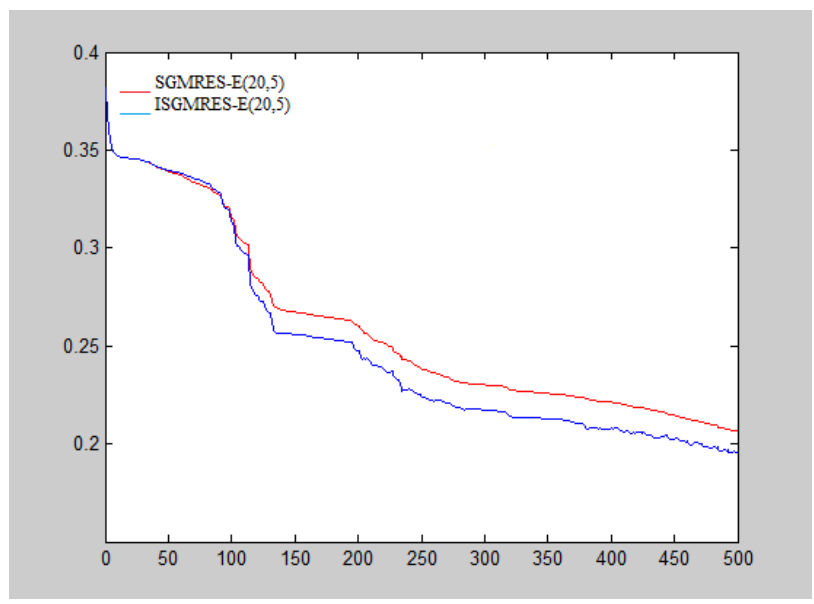

Figure 4: Comparison of convergence of SGMRES-E(m,k) and ISGMRES-E(m,k) on Tols1090 matrix with up to 500 iteration

In Table 3, SGMRES-DR(m,k) and ISGMRES-DR(m,k) is compared. According to Table 3,Figure5, and Figure 6, the performance of ISGMRES-DR $(\mathrm{m}, \mathrm{k})$ is better than SGMRES$\mathrm{DR}(\mathrm{m}, \mathrm{k})$.

Table 3: Comparison of the results of SGMRES-DR(m,k) and ISGMRES-DR(m,k)

\begin{tabular}{cccccccc}
\hline Problem & m & k & error & $\begin{array}{c}\text { SGMRES- } \\
\text { DR }\end{array}$ & \multicolumn{3}{c}{ ISGMRES-DR } \\
\hline Tols1090 & 20 & 5 & 0.3292 & 200 & 10 & 0.3 & iteration \\
Tols1090 & 20 & 5 & 0.0327 & 500 & 5 & 0.005 & $\mathbf{3 5 5}$ \\
Zenios & 30 & 10 & 0.9576 & 200 & 10 & 0.00005 & $\mathbf{1 8 2}$ \\
Zenios & 30 & 10 & 0.9575 & 500 & 10 & 0.0005 & $\mathbf{5 0 0}$ \\
Qh148 & 20 & 5 & 0.9758 & 200 & 5 & 0.04 & $\mathbf{1 6 4}$ \\
Qh148 & 20 & 5 & 0.9757 & 500 & 5 & 0.02 & $\mathbf{4 5 3}$ \\
\hline
\end{tabular}


Advanced Computational Intelligence: An International Journal (ACII), Vol.3, No.2, April 2016

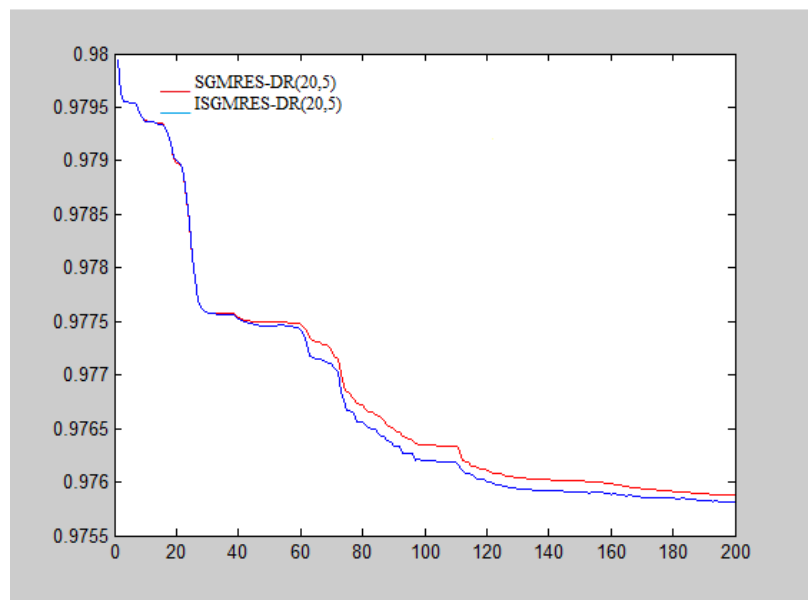

Figure 5: Comparison of convergence of SGMRES-DR(m,k) and ISGMRES-DR(m,k) on Qh148 matrix with up to 200 iteration

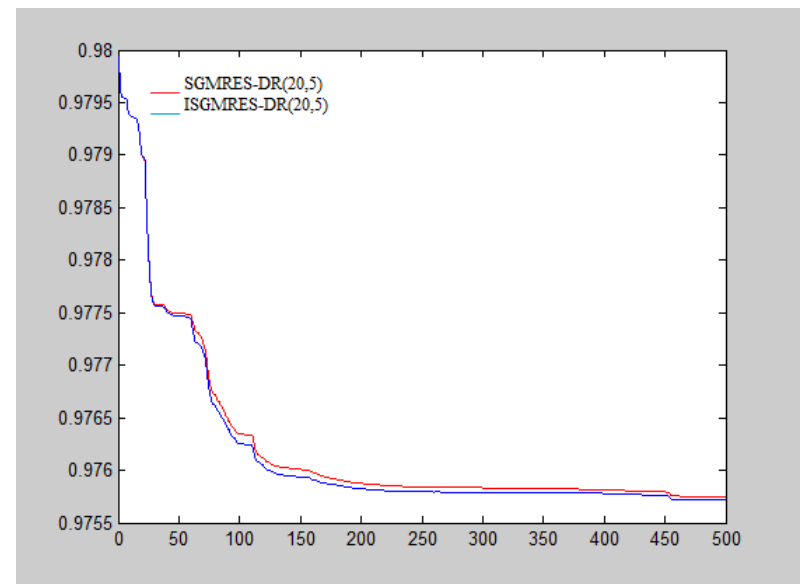

Figure 6: Comparison of convergence of SGMRES-DR(m,k) and ISGMRES-DR(m,k) on Qh148 matrix with up to 500 iteration

It should be noted that the computational complexity of each iteration of ISGMRES $(\mathrm{m})$, ISGMRES-E $(\mathrm{m})$, and ISGMRES-DR $(\mathrm{m})$ is approximately equal to each iteration of SGMRES(m), ISGMRES-E(m), and ISGMRES-DR(m), respectively.

As it can be observed from the above tables and figures, the proposed methods compared with corresponding standard methods for finding solutions with the same error require a lesser number of iterations.

\section{CONCLUSiOnS}

Based on the mathematical techniques, to improve the performance of SGMRES(m), SGMRES$\mathrm{E}(\mathrm{m}, \mathrm{k})$ and SGMRES-DR(m, k) methods, [5] - [8] - [7] - [13], that each of which have their own computational complexity and sometimes execution time and number of operations in the matrix vector multiplication greatly increase, a modified technique is proposed. Although, improving the 
efficiency of these algorithms by using the concepts and techniques of artificial intelligence has received little attention, in this paper, an intelligent heuristic method that has very little computational complexity is used to improve the performance of procedures SGMRES $(\mathrm{m})$, SGMRES-E $(\mathrm{m}, \mathrm{k})$ and SGMRES-DR(m, k).The numerical results obtained from implementation of the proposed method on several University of Florida standard matrixes confirm the efficiency of the proposed method.

\section{REFERENCES}

[1] Y. Saad, M.H. Schultz, (1986) GMRES: a generalized minimal residual algorithm for solving nonsymmetric linear systems, SIAM Journal on Scientific and Statistical Computing 7, 865-869.

[2] H.F. Walker, L. Zhou, (1994) A simpler GMRES, Numerical Linear Algebra with Applications 1, 571-581.

[3] Y. Saad, (2003) Iterative Methods for Sparse Linear Systems, 2nd ed., SIAM, Philadelphia.

[4] P. Jiránek, M. Rozlo`zník, M.H. Gutknecht, (2008) How to make simpler GMRES and GCR more stable, SIAM Journal on Matrix Analysis andApplications 30, 1483-1499.

[5] R.B. Morgan, (1995) A restarted GMRES method augmented with eigenvectors, SIAM Journal on MatrixAnalysis and Applications 16, 1154-1171.

[6] R.B. Morgan,(2000) Implicitly restarted GMRES and Arnoldi methods for nonsymmetric systems of equations, SIAM Journal on Matrix Analysisand Applications 21, 1112-1135.

[7] R.B. Morgan, (2002) GMRES with deflated restarting, SIAM Journal on Scientific Computing 24, 20-37.

[8] R.B. Lehoucq, D.C. Sorensen, (1996) Deflation techniques for an implicitly restarted Arnoldi iteration, SIAM Journal on Matrix Analysis and Applications 17, 789-821.

[9] R. Boojhawon, M. Bhuruth, (2004) Restartedsimpler GMRES augmented with harmonic Ritz vectors, Future Generation Computer Systems 20,389-397.

[10] R.B. Morgan, M. Zeng, (1998) Harmonic projection methods for large non-symmetric eigenvalue problems, Numerical Linear Algebra with Applications 5, 33-55.

[11] G.H. Golub, C.F.V. Loan ,(1996) Matrix Computations, 3rd ed., John Hopkins University Press, Baltimore, MD.

[12] S. Goossens, D. Roose, (1999) Ritz and harmonic Ritz values and the convergence of FOM and GMRES, Numerical Linear Algebra with Applications6, 281-293.

[13] Yiqin Lin, Liang Baob, Qinghua Wu. (2012)Simpler GMRES with deflated restarting, Mathematics and Computers in Simulation 82, 2238-2252. 